\title{
Boundary conditions for integrable equations
}

\author{
V Adler†, B Gurel“ ‡, M Gurses”キ and I Habibullin $†$ \\ † Ufa Institute of Mathematics, Russian Academy of Sciences, Chernyshevsky str. 112, 450000 \\ Ufa, Russia \\ ‡ Department of Mathematics, Faculty of Science, Bilkent University, 06533 Ankara, Turkey
}

Received 9 August 1996, in final form 6 December 1996

\begin{abstract}
The problem of constructing boundary conditions for nonlinear equations compatible with higher symmetries is considered. In particular, this problem is discussed for the sineGordon, Jiber-Shabat, Liouville and KdV equations. New results are obtained for the last two ones. The boundary condition for the KdV contains two arbitrary constants. The substitution $\mathrm{u}=$ $\mathrm{q}_{\mathrm{x}}$ maps it onto the boundary condition with linear dependence on $\mathrm{t}$ for the potentiated $\mathrm{KdV}$.
\end{abstract}

\section{Introduction}

Applications of classical Lie symmetries to boundary value problems are well known [1]. In contrast, the question of involving higher symmetries in the same problem has received much less attention, unlike, say, the Cauchy problem. However, it is now well understood that the approach of higher symmetries has become the basis of modern integrability theory [2]. A number of attempts to apply the inverse scattering method (ISM) to the initial boundary value problem have been made. It turned out that if both the initial data and boundary value are chosen arbitrarily, then the ISM essentially loses its power. On the other hand, the investigation by Sklyanin [3] based on the R-matrix approach demonstrated that there are certain boundary conditions that are completely compatible with integrability. The analytical aspects of such kind of problems were studied in [4]. It later became clear that the boundary value problems found can effectively be investigated with the help of the Backlund transformation [6]."

Below we will discuss a higher symmetry test, proposed in $[7,8]$ to verify whether the boundary condition given is compatible with the integrability property of the equation. It is worth noting that all known classes of boundary conditions that are compatible with integrability pass this symmetry test. In [8-10] we gave an approach for finding the boundary conditions compatible with infinitely many higher symmetries. Boundary conditions involving explicit time dependence for the Toda lattice compatible with higher symmetries have recently been studied in [11]. It was observed that finite-dimensional systems obtained from the Toda lattice by imposing boundary conditions consistent with symmetries are closely connected with Painleve-type equations.'

In previous works [8-10] we have mostly considered boundary conditions compatible with homogeneous symmetries. In this work we extend some of our previous results by utilising inhomogeneous symmetries. We show that for some integrable equations this 
extension changes the boundary conditions found previously. As an example, the boundary conditions $\mathrm{u}=$ constant and $\mathrm{u}_{\mathrm{xx}}=$ constant found in this work for the $\mathrm{KdV}$ equation

0305-4470/97/103505+09\$19.50 (C)1997 IOP Publishing Ltd

(see the proposition in section 3) are new. Notice that these boundary conditions found in a formal algebraic way turned out to have nice analytic properties. Recently in [12] it was shown that the KdV equation admits regular finite-gap solutions with these boundary conditions.

\section{Symmetry-compatible boundary conditions}

Before presenting our results we give a brief review of our previous work $[7,8,10]$. Let an integrable nonlinear partial differential equation and a set of boundary conditions be given by

$$
\mathrm{u}_{\mathrm{t}}=\left.\mathrm{f}\left(\mathrm{u}, \mathrm{u}_{1}, \mathrm{u}_{2}, \ldots, \mathrm{u}_{\mathrm{n}}\right) \quad(1) \mathrm{p}\left(\mathrm{u}, \mathrm{u}_{1}, \mathrm{u}_{2}, \ldots, \mathrm{u}_{\mathrm{k}}\right)\right|_{\mathrm{x}=\mathrm{x}_{0}}=0 \quad(2)
$$

where $\mathrm{p}=\left(\mathrm{p}_{1}, \ldots, \mathrm{p}_{\mathrm{s}}\right)$ is a vector function, $\mathrm{s}<\mathrm{n}$. Here $\mathrm{u}_{\mathrm{i}}$ stands for the partial derivative of order i with respect to the variable $x$. Suppose that equation (1) possesses a higher symmetry

$$
\mathrm{u}_{\tau}=\mathrm{g}\left(\mathrm{u}, \mathrm{u}_{1}, \ldots, \mathrm{u}_{\mathrm{m}}\right) .
$$

We call the problem (1), (2) compatible with the symmetry (3) if for any initial data prescribed at the point $\mathrm{t}=0$, a common solution to equations (1), (3) exists satisfying the boundary condition (2). Let us explain more exactly what we mean. Evidently, one can only differentiate the constraint (2) with respect to the variables $t$ and $\tau$ (but not with respect to $x$ ). For instance, it follows from (2) that

$$
\sum_{i=0}^{n} \frac{\partial p}{\partial u_{i}}\left(u_{i}\right)_{\tau}=
$$

where one should replace $\tau$-derivatives by means of equation (3).

Definition. The boundary value problem (1), (2) is called compatible with the symmetry (3) if equation (4) holds identically by means of the condition (2) and its consequences obtained by differentiation with respect to $t$.

To formulate an effective criterion of compatibility of the boundary value problem with a symmetry it is necessary to introduce some new set of dynamical variables consisting of the vector $\mathrm{v}=\left(\mathrm{u}, \mathrm{u}_{1}, \mathrm{u}_{2}, \ldots, \mathrm{u}_{\mathrm{n}}-1\right)$ and its $\mathrm{t}$-derivatives: $\mathrm{v}_{\mathrm{t}}, \mathrm{v}_{\mathrm{tt}}, \ldots$. Passing to this set of variables allows one really to exclude the dependence on the variable $\mathrm{x}$. In terms of these variables the symmetry (3) and the constraint (2) take the form

$$
\begin{gathered}
v^{\tau}=G\left(\boldsymbol{v}, \boldsymbol{v}_{t}, \boldsymbol{v}_{t t}, \ldots, \begin{array}{c}
\partial^{m_{1}} \boldsymbol{v} \\
\partial t^{m_{1}}
\end{array}\right) \\
P\left(\boldsymbol{v}, \frac{\partial \boldsymbol{v}}{\partial t}, \ldots, \frac{\partial^{k_{1}} \boldsymbol{v}}{\partial t^{k_{1}}}\right)=0
\end{gathered}
$$

The following criterion of compatibility was established in [8].

Theorem 1. The boundary value problem (1), (2) is compatible with the symmetry (3) if and only if the differential constraint (6) is consistent with the system (5). 
We call the boundary condition (2) compatible with the integrability property of equation (1) if the problem (1), (2) is compatible with an infinite series of linearly independent higherorder symmetries.

The motivation for such a definition originated with the widely studied examples of boundary value problems for nonlinear equations (see $[3,4,13]$ ) consistent with the inverse scattering transform method.

Our main observation is that if the boundary condition is compatible with one higher symmetry then as a rule it is compatible with an infinite number of symmetries which form a set $\mathrm{S}$ with infinite elements. The set $\mathrm{S}$ may or may not contain the whole symmetries of (1). For instance, $\mathrm{S}$ contains the even-ordered time-independent symmetries for the Burgers equation.

We suppose that equation (1) admits a recursion operator (definition of integrability of (1)) of the form (see $[2,14,15])$

$$
R=\sum_{i=0}^{i_{1}} \alpha_{i} D^{i}+\sum_{i=0}^{k_{1}} \alpha_{-1, i} D^{-1} \alpha_{-2, i} \quad \mathrm{i}_{1}>0 \quad \mathrm{k}_{1}>0
$$

where $\alpha_{\mathrm{i}}, \alpha-1, \mathrm{i}, \alpha-2, \mathrm{i}$ are functions of the dynamical variables, $\mathrm{D}$ is the total derivative with respect to $\mathrm{x}$. Recursion operator when applied to a symmetry produces new symmetries. Passing to the new dynamical variables $v_{,}, v_{t}, v_{t}, \ldots$, one can obtain, from (7), the recursion operator $\mathbf{R}$ of the system of equations (5):

$$
\mathbf{R}_{\mathrm{i}=0}^{\mathrm{M}} \mathrm{X} \mathrm{a}_{\mathrm{i}}\left(\partial_{\mathrm{t}}\right)^{\mathrm{i}+} \mathrm{X}_{\mathrm{i}=0}^{\mathrm{K}-1, \mathrm{i}\left(\partial_{\mathrm{t}}^{-1}\right) \mathrm{a}-2, \mathrm{i}} \quad \mathrm{M}>0 \quad \mathrm{~K}>0
$$

where the coefficient matrices $a_{i},{ }_{-1, i},{ }^{a}{ }_{-2, i}$ depend on $v$ and on a finite number of its $t-$ derivatives, and $\partial_{t}$ is the operator of the total derivative with respect to t. If (1) is a scalar equation, then $\mathbf{R}$ is a scalar operator and $\mathbf{R}$ is an $\mathrm{n} \times \mathrm{n}$ matrix-valued operator. Our further considerations are based on the following propositions, which really affirm that if an equation admits a differential connection, then an infinite number of its higher symmetries admits also the same connection. These are propositions 2.2 and 2.1 of [10], respectively.

Theorem 2. Suppose that $\mathrm{p}(\mathrm{v})=0$ is set of $\mathrm{n}-1$ functionally independent constraints and that there exists a positive integer $\mathrm{n}_{0}$ such that the coefficient matrix $b_{N}$ in the expression

Rcompatible with the symmetryn $=b_{N}\left(\partial^{0}\right)_{N}+b_{N-1}(\partial t)_{N-1}+\cdots \sigma_{\tau}=$ is proportional to the identity matrix. Then $\boldsymbol{R}_{n_{0}} \sigma_{t}$ if and only if it is compatible with the $\operatorname{symmetryp}(v)=0$ is

$\sigma_{\tau}=\mathrm{H}\left(\mathbf{R}^{\mathrm{n}}\right) \sigma_{\mathrm{t}}$, where $\mathrm{H}$ is a scalar polynomial with constant coefficients, and $\sigma=\mathrm{v}^{\mathrm{T}}$.

This theorem plays a cruical role in determining the test symmetry $\mathbf{R}^{\mathrm{n}_{0}} \sigma_{\mathrm{t}}$ (or the positive integer $\mathrm{n}_{0}$ ) which is going to be the first element of S. Boundary conditions found with the aid of this theorem will in general be compatible with only homogenous higher symmetries. Hence they will not be most general symmetry-compatible boundary conditions. For this purpose we have the following theorem. 
Theorem 3. Suppose that $\mathrm{p}(\mathrm{v})=0$ is a set of $\mathrm{n}-1$ functionally independent constraints. If $\mathrm{p}(\mathrm{v})$

$=0$ is compatible with the symmetry $\sigma_{\tau}=\mathrm{T}(\mathbf{R}) \sigma_{\mathrm{t}}$ then it is compatible with every symmetry

$\sigma_{\tau}=\mathrm{H}(\mathrm{T}(\boldsymbol{R})) \sigma_{\mathrm{t}}$ where $\mathrm{T}$ and $\mathrm{H}$ are arbitrary scalar polynomials with constant coefficients.

The problem of the classification of integrable boundary conditions is solved completely for the Burgers equation (see [8])

$$
\mathrm{u}_{\mathrm{t}}=\mathrm{u}_{2}+2 \mathrm{uu}_{1} .
$$

Theorem 4. If the boundary condition $\left.\mathrm{p}\left(\mathrm{u}, \mathrm{u}_{1}\right)\right|_{\mathrm{x}=\mathrm{x}_{0}}=0$ is compatible at least with one higher symmetry of the Burgers equation (9) then it is compatible with all even-order homogeneous symmetries and is of the form $c_{1}\left(u_{1}+u^{2}\right)+c_{2} u+c_{3}=0$.

\section{The KdV equation}

Very often the simplest integrable boundary condition can be found by the use of the reflection-type symmetry like $\mathrm{x} \rightarrow-\mathrm{x}, \mathrm{u} \rightarrow \mathrm{h}(\mathrm{u})$. It is unexpected that equations which do not admit any reflection symmetry nevertheless admit boundary conditions compatible with integrability. Let us consider, for instance, the famous $\mathrm{KdV}$ equation

$$
\mathrm{u}_{\mathrm{t}}=\mathrm{u}_{\mathrm{xxx}}+6 \mathrm{uu}_{\mathrm{x}} \text {. }
$$

As was established in [8], the $\mathrm{KdV}$ equation admits the homogeneous boundary condition

$$
\mathrm{u}=0 \quad \mathrm{u}_{\mathrm{xx}}=0 \quad \mathrm{x}=\mathrm{x}_{0} .
$$

A further generalization of the above boundary condition is as follows:

$$
\mathrm{u}=\mathrm{c} \quad \mathrm{u}_{\mathrm{xx}}=-3 \mathrm{c}^{2} \quad \mathrm{x}=\mathrm{x}_{0}
$$

where $\mathrm{c}$ is an arbitrary constant. Note that the boundary conditions (11) and (12) consist of two independent scalar constraints and, as is proved in [8], are compatible with all homogeneous symmetries of orders divisible by three and also with every symmetry from the linear envelope of symmetries divisible by three (see below).

One of the principal problems here is to find the set $S$ and to identify the simplest symmetries from $\mathrm{S}$ which can be considered as test symmetries. Due to the theorems 2 and 3, boundary conditions compatible with a test symmetry will be compatible with the all elements of the set S. It is evident, for instance, that to describe all boundary conditions for the Burgers equation consistent with integrability it is sufficient to examine only one test symmetry, namely the fourth-order homogeneous symmetry:

$$
u_{\tau_{2}}=u_{x x x x}+4 u u_{x x x}+10 u_{x} u_{x x}+6 u^{2} u_{x x}+12 u u_{x}^{2}+4 u^{3} u_{x} .
$$

All boundary conditions compatible with this symmetry will be compatible with all even symmetries of the Burgers equation. In [11] it was shown that the Toda lattice is served by a whole family of test symmetries depending on a numerical parameter. So actually the set $\mathrm{S}$ may depend on the parameters.

Starting with this observation and with the boundary condition (12) for which the test symmetry coincides with the ninth-order homogeneous one, we now use theorem 3 . As a test symmetry, in this case we take a linear combination of all symmetries of the KdV equation of both types either higher or classical with undefined coefficients. Thus we have seven summands: classical symmetries corresponding to Galilean invariance, $\mathrm{x}$-translation, $\mathrm{t}$ - 
translation, scaling and three higher-order symmetries, fifth, seventh and ninth. First let us give the ninth-order higher symmetry of the KdV equation:

$u_{\tau 3}=f_{9}=u_{t t t}+96 u^{3} u_{t}+54 u^{4} v+18 u^{2} w_{t}+36 u^{2} v w+36 u u_{t} W$

$$
-6 u_{t} v^{2}+6 w w_{t}+6 v w^{2}
$$

written in terms of the new dynamical variables $\mathrm{u}, \mathrm{v}=\mathrm{u}_{\mathrm{x}}, \mathrm{w}=\mathrm{u}_{\mathrm{xx}}$ and their t-derivatives.

Now we consider the inhomogeneous symmetry

$u_{\tau_{3}^{\prime}}=f_{9}^{\prime}=f_{9}+a_{1} f_{7}+a_{2} f_{5}+a_{3} f_{3}+a_{4} f_{1}+a_{5 \text { (scale symmetry) }}$

$+\mathrm{a}_{6}$ (Galilean symmetry)

where $f_{j}$ denotes the jth-order symmetry of the KdV equation, for each $j$, and in particular $f_{1}=$ $u_{x}=v$. Using $u_{x}=v$ and $u_{x x}=w$, we then get the following system of equations from (15) by differentiating it with respect to $\mathrm{x}$ :

$u_{\tau 30}=u_{\tau 3}+a_{1}\left(v_{t t}+22 u^{2} u_{t}+2 u w_{t}+4 w_{t}+4 u v w\right.$

$$
\begin{aligned}
& \left.+8 \mathrm{u}^{3} \mathrm{v}-2 \mathrm{v}^{3}\right)+\mathrm{a}_{2}\left(\mathrm{w}_{\mathrm{t}}+4 \mathrm{uu}_{\mathrm{t}}+2 \mathrm{v}\left(\mathrm{w}+3 \mathrm{u}^{2}\right)\right)+\mathrm{a}_{3} \mathrm{u}_{\mathrm{t}}+\mathrm{a}_{4} \mathrm{v} \\
& +\mathrm{a}_{5}\left(3 \mathrm{tu}_{\mathrm{t}}+\mathrm{xv}+2 \mathrm{u}\right)+\mathrm{a}_{6}(1+6 \mathrm{tv}) \mathrm{vt30}=
\end{aligned}
$$

$\mathrm{v} \tau_{\tau}+\mathrm{a}_{1}\left(\mathrm{Wtt}_{\mathrm{tt}}+2 \mathrm{uutt}_{\mathrm{t}}+2 \mathrm{vWt}+4 \mathrm{vtw}+12 \mathrm{uvut}\right.$

$$
\left.+10 u^{2} v_{t}+4 u^{2} t+8 u^{3} w+4 u w^{2}-2 v^{2} w\right)+a_{2}\left(u_{t t}-2 u v_{t}\right.
$$

$$
\left.+2 w\left(w+3 u^{2}\right)\right)+a_{3} v_{t}+a_{4} w+a_{5}\left(3 t v_{t}+x w+3 v\right)+6 a_{6} t w
$$

$\mathrm{w}_{\tau 30}=\mathrm{w}_{\tau 3}+\mathrm{a}_{1}\left(\mathrm{uttt}_{\mathrm{tt}}-4 \mathrm{uvtt}_{\mathrm{tt}}-2 \mathrm{vutt}_{\mathrm{tt}}-4 \mathrm{uvvt}\right.$

$$
\begin{aligned}
& -2 v^{2} u_{t}+6 w w_{t}+8 u^{3} u_{t}+10 u^{2} w_{t}+20 u w u_{t}-48 u^{4} v \\
& \left.-24 u^{2} v w+12 u v^{3}\right)+a_{2}\left(v_{t t}-2 v_{t}-2 u w_{t}+4 w u_{t}-12 u v w\right. \\
& \left.+6 u^{2} u_{t}-36 u^{3} v\right)+a_{3} w_{t}+a_{4}\left(u_{t}-6 u v\right)+a_{5}\left(3 t w_{t}\right. \\
& \left.+x u_{t}-6 x u v+4 w\right)+a_{6}\left(6 u_{t}-36 t u v\right)
\end{aligned}
$$

where $\mathrm{u}_{\tau \mathrm{j}}=\mathrm{f}_{3+2 \mathrm{j}}, \forall \mathrm{j}=-1,0,1,2, \ldots, \mathrm{u}_{\tau 3}$ is given in (14) and $\mathrm{v}_{\tau 3}=\mathrm{D}_{\mathrm{x}} \mathrm{u}_{\tau 3}, \mathrm{w}_{\tau 3}=\mathrm{D}_{\mathrm{x}} \mathrm{v}_{\tau 3}$. The boundary conditions with one constraint are not compatible with the higher symmetries. Hence we now look for boundary conditions with two constraints $\mathrm{p}_{1}(\mathrm{u}, \mathrm{v}, \mathrm{w})=0, \mathrm{p}_{2}(\mathrm{u}, \mathrm{v}, \mathrm{w})=0$ compatible with the above system. Here we have the following distinct types of these constraints:

(i) $\mathrm{u}=\mathrm{h}_{1}(\mathrm{w}), \mathrm{v}=\mathrm{h}_{2}(\mathrm{w})$,

(ii) $\mathrm{v}=\mathrm{h}_{1}(\mathrm{u}), \mathrm{w}=\mathrm{h}_{2}(\mathrm{u})$,

(iii) $\mathrm{u}=\mathrm{h}_{1}(\mathrm{v}), \mathrm{w}=\mathrm{h}_{2}(\mathrm{v})$. 
Direct calculations lead to the following:

Proposition. The constraints

$$
\mathrm{u}=\mathrm{k}_{1} \quad \mathrm{u}_{\mathrm{xx}}=\mathrm{k}_{2} \quad \mathrm{x}=\mathrm{x}_{0}
$$

are integrable boundary conditons for the $\mathrm{KdV}$ equation where $\mathrm{k}_{1}$ and $\mathrm{k}_{2}$ are arbitrary constants and the non-zero constant coefficients in the system (16) are given by $\mathrm{a}_{2}=-6 \mathrm{~m}$ and $\mathrm{a}_{4}=6 \mathrm{~m}^{2}$ with $^{m}=3 k_{1}^{2}+k_{2}$. The boundary conditions (17) are compatible with a set of infinitely many higher symmetries by theorem 3 .

Note that the symmetry (15) contains the classical symmetry $f_{1}$ as a summand, and because of this one cannot use theorem 3 immediately. On the other hand some of the higher symmetries with which the boundary condition (17) are compatible, are, for instance:

$$
\mathrm{u}_{\tau 60}=\mathrm{f}_{15}-10 \mathrm{mf} 11+30 \mathrm{~m} 2 \mathrm{f} 7
$$

and, applying theorem 3, the boundary conditions will be compatible with an infinite set of symmetries. Further calculations show that the above boundary conditions are also compatible with the following higher inhomogeneous symmetry containing the term $\mathrm{f}_{1}$ :

$$
\mathrm{u}_{\tau 90}=\mathrm{f}_{21}-14 \mathrm{mf} 17+70 \mathrm{~m}_{2} \mathrm{f}_{13}-770 \mathrm{~m} 4 \mathrm{f} 5+868 \mathrm{~m}_{5} \mathrm{f}_{1} .
$$

The boundary conditons given in the above proposition are the most general ones. Previously [8-10] we had $\mathrm{k}_{1}=\mathrm{k}_{2}=0$.

Now let us consider the potentiated $\mathrm{KdV}$ equation

$$
q_{t}=q_{x x x}+3 q_{x}^{2}
$$

which is connected to (10) by the formula $u=q_{x}$. It is easy to check that this substitution maps the boundary condition (17) onto the boundary condition with explicit dependence on t:

$$
\mathrm{q}=\mathrm{kt}+\mathrm{c} \quad \mathrm{q}_{\mathrm{x}}=\mathrm{a} \quad \mathrm{x}=\mathrm{x}_{0}
$$

the compatibility of which with integrability can also be checked directly. It is consistent with the symmetries

$$
\begin{aligned}
& \mathrm{q}_{\tau}=\mathrm{f}_{3}-\mathrm{kf}_{-1} \quad \mathrm{q}_{\tau}=\mathrm{f}_{9}-6 \mathrm{kf}_{5}+6 \mathrm{k}^{2} \mathrm{f}_{1} \mathrm{q}_{\tau}=\mathrm{f}_{15}- \\
& 10 \mathrm{kf}_{11}+30 \mathrm{k}^{2} \mathrm{f}_{7}-25 \mathrm{k}^{4} \mathrm{f}_{-1} \quad \ldots
\end{aligned}
$$

where the $q_{t j}=f_{j}$ are the homogeneous symmetries rewritten in terms of the variable $q$ and $f_{-1}$ $=1$.

Another example of boundary conditions with explicit $t$ dependence can be obtained by the known point transformation which maps the $\mathrm{KdV}$ equation (10) onto the cylindirical $\mathrm{KdV}$ equation

$$
u_{t}=u_{x x x}+6 u u_{x}-\frac{u}{2 t} .
$$

The boundary conditions take the form

$$
\left.u\right|_{x=x_{0}}=\left.\frac{A}{t} \quad u_{x x}\right|_{x=x_{0}}=\frac{B}{t^{2}}
$$

where A, B are arbitrary constants. 
Finally, the integrable boundary condition for the mKdV equation

$$
\begin{aligned}
& \mathrm{ut}=\mathrm{u}_{\mathrm{xxx}}+6 \mathrm{u}_{2} \mathrm{u}_{\mathrm{x}} \\
& \mathrm{u}=0 \quad \mathrm{u}_{\mathrm{x}}=\mathrm{a}
\end{aligned}
$$

is compatible with the symmetries $\quad \mathrm{x}=\mathrm{x}_{0}$

$$
\begin{aligned}
& u \tau=f 9+6 a 2 f_{5}+6 a 4 f_{1} \quad u \tau=f_{15}+10 a 2 f_{11}+30 a 4 f_{7} u_{\tau}=f_{21}+ \\
& 14 a^{2} f_{17}+70 a^{4} f_{13}-770 a^{8} f_{5}-868 a^{10} f_{1} \quad \ldots
\end{aligned}
$$

Of course this result can be obtained by use of the Miura map.

\section{Hyperbolic-type equations}

The definition of the integrable boundary condition remains the same for hyperbolic-type equations. The only difference is that in this case the higher symmetry contains partial derivatives with respect to both independent variables $t$ and $x$. Let us describe, for example, the boundary value problems of the form

$$
\mathrm{p}\left(\mathrm{u}, \mathrm{u}_{\mathrm{x}}\right)=0 \mathrm{x}=\mathrm{x}_{0} \quad(19) \mathrm{u}_{\mathrm{tt}}-\mathrm{u}_{\mathrm{xx}}+\sin \mathrm{u}=0
$$

for the sine-Gordon equation, which are compatible with the third-order symmetry.

It was shown in [16] that the complete algebra of higher symmetries for this equation, i.e. $\mathrm{u}_{\xi \eta}=\mathrm{sinu}$, where $2 \xi=\mathrm{x}+\mathrm{t}, 2 \eta=\mathrm{x}-\mathrm{t}$ splits into the direct sum of two algebras consisting of the symmetries of the equations $u_{\tau}=u_{\xi \xi \xi}+u_{\xi}^{3} / 2, u_{\tau}=u_{\eta \eta \eta}+u_{\eta}^{3} / 2$, respectively, which are quite simply the potentiated $\mathrm{mKdV}$ equation. In particular, the following flow commutes with the sine-Gordon equation:

$$
u_{\tau}=c_{1}\left(u_{\xi} \xi \xi+u_{3} \xi / 2\right)+c_{2}\left(u_{\eta \eta \eta}+u_{3 \eta} / 2\right)
$$

It can easily be verified that the symmetry (21) is compatible with the boundary condition of the form (19) only if constraint holds $c_{1}=-c_{2}$. Under this constraint the symmetry is rewritten in original variables as the system

$$
\begin{aligned}
& \mathrm{u} \tau=8 \mathrm{uttt}+6 \mathrm{ut} \cos \mathrm{u}+3 \mathrm{w} 2 \mathrm{ut}+\mathrm{u} 3 \mathrm{t} \\
& \mathrm{W} \tau=8 \mathrm{Wttt}+6 \mathrm{Wt} \cos \mathrm{u}+6 \mathrm{uttwut}+3 \mathrm{w} 2 \mathrm{Wt}+3 \mathrm{u} 2 \mathrm{t} \mathrm{wt} .
\end{aligned}
$$

where $\mathrm{w}=\mathrm{u}_{\mathrm{x}}$. According to the theorem 1 above one reduces the problem of finding integrable boundary conditions to the problem of looking for differential connections admissible by this system. Straightforward calculations prove that the boundary condition (19) has to have one of the forms

$$
\mathrm{u}=\text { constant } \quad \mathrm{u}_{\mathrm{x}}+\mathrm{a} \cos (\mathrm{u} / 2)+\mathrm{b} \sin (\mathrm{u} / 2)=0 \quad \mathrm{x}=\mathrm{x}_{0} .
$$

Note that the list of boundary conditions (23) coincides with that found by Zamolodchikov within the framework of the R-matrix approach [17]. The particular cases of (23) were studied earlier in $[3,5]$. The compatibility of the first boundary condition in (23) with the usual version of ISM was declared earlier in [5]. However, the statement was based on a mistake (see [13]). Our requirement of consistency is weaker than that used in [5]. Applications of 
these and similar problems for the sine-Gordon equation and the affine Toda lattice in the quantum field theory are studied in [18-20].

One can prove that the boundary conditions (23) are compatible with a rather large subclass of symmetries of the sine-Gordon equation such that

$$
\mathrm{u}_{\tau}=\varphi\left(\mathrm{u}, \mathrm{u}_{1}, \ldots \mathrm{u}_{\mathrm{k} 1}\right)-\varphi\left(\mathrm{u}_{1} \mathrm{u}_{1}, \ldots . \mathrm{u}_{\mathrm{k} 1}\right)-
$$

where $\mathrm{u}_{\mathrm{j}}=\partial^{\mathrm{j}} \mathrm{u} / \partial \xi^{\mathrm{j}}, \mathrm{u}_{\mathrm{j}}=\overline{\partial^{\mathrm{j}} \mathrm{u}} / \partial \eta^{\mathrm{j}}$, and equation $\mathrm{u}_{\tau}=\varphi_{\mathrm{i}}\left(\mathrm{u}, \mathrm{u}_{1}, \ldots \mathrm{u}_{\mathrm{ki}}\right)$ is a symmetry of equation $\mathrm{u}_{\tau}=$ $u_{\xi \xi \xi}+u^{3} \xi / 2$ for $i=1,2$.

Another well known integrable hyperbolic-type equation

$$
\mathrm{u}_{\mathrm{tt}}-\mathrm{u}_{\mathrm{xx}}=\exp (\mathrm{u})+\exp (-2 \mathrm{u})
$$

has applications in the geometry of surfaces. It was first found by Tzitzeica [21]. The presence of higher symmetries for this equation has been established by Jiber and Shabat [16]. The simplest higher symmetry of this equation is of fifth order:

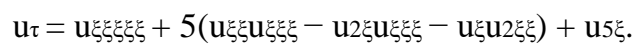

It was proved in [22] that the higher symmetry algebra for (25) is the direct sum of the higher symmetry algebras of (26) and of equation obtained from (26) by replacing $\xi$ by $\eta$.

Let us look for boundary conditions of the form

$$
\mathrm{p}\left(\mathrm{u}, \mathrm{u}_{\mathrm{x}}\right)=0 \quad \mathrm{x}=\mathrm{x}_{0}
$$

for equation (25) that are compatible with the symmetry $\mathrm{u}_{\tau}$

$$
\begin{aligned}
& =u \xi \xi \xi \xi \xi+5(u \xi \xi u \xi \xi \xi-u 2 \xi u \xi \xi \xi-u \xi u \xi \xi 2)+u 5 \xi-u \eta \eta \eta \eta \eta \\
& -5\left(u_{\eta \eta} u_{\eta \eta \eta}-u_{2 \eta} u_{\eta \eta \eta}-u_{\eta} u 2 \eta \eta\right)-u 5 \eta .
\end{aligned}
$$

Rather simple but tediously long computations lead to the following statement [23].

Theorem 5. The boundary conditions (27) for the Jiber-Shabat equation compatible with the symmetry (28) (and then compatible with integrability) are either of the form

$\mathrm{u}_{\mathrm{x}}+\left.\mathrm{a} \exp (-\mathrm{u})\right|_{\mathrm{x}=\mathrm{x}_{0}=0} \quad$ or $\quad \mathrm{u}_{\mathrm{x}}+\mathrm{a} \exp (\mathrm{u} / 2) \pm\left.\exp (-\mathrm{u})\right|_{\mathrm{x}=\mathrm{x}_{0}}=0$

where $\mathrm{a}$ is arbitrary.

The famous Liouville equation $\mathrm{u}_{\mathrm{tt}}$

$$
-u_{x x}+\exp (u)=0
$$

admits the rather rich symmetry algebra (see [16]). Let us take as a test symmetry the following simplest one:

$$
u_{\tau}=8 u_{t t t}+u_{t}\left(6 \exp (u)-3 u_{x}^{2}-u_{t}^{2}\right) .
$$

Passing to the appropriate set of dynamical variables $\mathrm{u}, \mathrm{w}=\mathrm{u}_{\mathrm{x}}, \mathrm{u}_{\mathrm{t}}, \mathrm{w}_{\mathrm{t}}, \ldots$, one obtains the system of the form 


$$
\begin{aligned}
& \mathrm{u}_{\tau}=8 \mathrm{u}_{\mathrm{ttt}}+\mathrm{u}_{\mathrm{t}}\left(6 \exp (\mathrm{u})-3 \mathrm{w}^{2}-\mathrm{u}_{\mathrm{t}}^{2}\right) \mathrm{w}_{\tau}=8 \mathrm{w}_{\mathrm{ttt}}+ \\
& \mathrm{w}_{\mathrm{t}}\left(6 \exp (\mathrm{u})-3 \mathrm{w}^{2}\right)-3 \mathrm{u}_{\mathrm{t}}\left(\mathrm{w}_{\mathrm{t}} \mathrm{u}_{\mathrm{t}}+2 \mathrm{wu}_{\mathrm{tt}}\right)
\end{aligned}
$$

where $w=u_{x}$. It is not difficult to find boundary conditions compatible with this symmetry:

$$
\mathrm{u}_{\mathrm{x}}+\mathrm{a} \exp (-\mathrm{u} / 2)+\left.\mathrm{b} \exp (\mathrm{u} / 2)\right|_{\mathrm{x}=\mathrm{x}_{0}}=0
$$

or

$$
\mathrm{u} \mathrm{x}_{\mathrm{x}=\mathrm{x}_{0}}=\text { constant }
$$

\section{Conclusion}

In the search for symmetry-compatible boundary conditions [8-10] of the integrable nonlinear partial differential equations we had so far used homogeneous generalized symmetries. In this work we showed that one may obtain more general boundary conditions if inhomogeneous generalized symmetries are used.

We applied the technique developed in our previous work [8-10] to the Burgers, $\mathrm{KdV}$, $\mathrm{mKdV}$ and hyperbolic-type equations by the use of inhomogeneous generalized symmetries. The boundary conditions obtained for Burgers remain the same. This confirms our uniqueness theorem for the boundary conditions of the Burgers equation [10]. The boundary conditions found here for the $\mathrm{KdV}$ and $\mathrm{mKdV}$ equations are new and more general than our previous results. In this paper we also included a new equation: the cylindrical KdV equation. The boundary conditions for this equation are time dependent. In the case of hyperbolic-type equations only the boundary conditions of the Liouville equation are new. The boundary conditions for others like sine-Gordon and Jiber-Shabat remain the same. The use of inhomogeneous generalized symmetries does not provide us with new boundary conditions.

\section{Acknowledgments}

This work has been supported by Russian Foundation for Fundamental Research and by the Turkish Scientific and Technical Research Council (TUBITAK). MG is an associate member of Turkish Academy of Sciences (TUBA).

\section{References}

[1] Ovsyannikov L V 1978 Group Analysis of Differential Equations (Moscow: Nauka)

[2] Mikhailov A V, Sokolov V V and Shabat A B 1991 What is Integrability? ed V E Zakharov (Berlin: Springer) p 115

[3] Sklyanin E K 1987 Funct. Anal. Prilozh. 2186

[4] Bibikov P N and Tarasov V O 1989 Teor. Mat. Fiz. 79334

[5] Bikbaev R F and Tarasov V O 1991 Algebra i Analiz 3 (4) 78

[6] Habibullin I T 1991 Matem. Zametki 494

[7] Habibullin I T 1993 Phys. Lett. 178A 369

[8] Gurel B, G“ urses M and Habibullin I 1994“'Phys. Lett. 190A 231

[9] Gurel T B, G" urses M and Habibullin I 1996 Integrable boundary conditions for evolution equations" Proc. Workshop on Nonlinear Physics: Theory and Experiment (Lecce, 1995) ed E Alfinito, M Boiti, L Martina and F Pempinelli (Singapore: World Scientific) p 131

[10] Gurel B, G“ urses M and Habibullin I 1995“ J. Math. Phys. 366809

[11] Adler V E and Habibullin I T 1995 J. Phys. A: Math. Gen. 286717 
[12] Adler V E, Habibullin I T and Shabat A B 1997 Boundary value problem for the KdV equation on half line Teor. Mat. Fiz. 11098

[13] Habibullin I T 1993 Teor. Mat. Phys. 96109

[14] Gurses M and Karasu A 1995" J. Math. Phys. 363485

[15] Fokas A S 1980 J. Math. Phys. 211318 Fokas A S 1987 Stud. Appl. Math. 77253

[16] Jiber A and Shabat A 1979 Dokl. Akad. Nauk SSSR 2471103

[17] Ghoshal S and Zamolodchikov A B 1993 Boundary state and boundary S matrix in two-dimensional integrable field theory Preprint RU-93-20, hep-th/9306002

[18] Saleur H, Skorik S and Warner N P 1994 The boundary sine-Gordon theory Preprint USC-94-013, hepth/9408004

[19] Corrigan E, Dorey P E, Rietdijk R H and Sasaki R 1994 Affine Toda field theory on a half-line Preprint hepth/9404108

[20] Bowcock P, Corrigan E and Rietdjk R H 1995 Background field boundary conditions for affine Toda field theories Preprint hep-th/9510071

[21] Tzitzeica G 1910 CR Acad. Sci. Paris 150955

[22] Jiber A V 1994 Symmetries and integrals of nonlinear equations Doctor of Science Thesis Ekaterinburg, Russia (in Russian)

[23] Habibullin I 1995 Symmetry approach in boundary value problems Preprint solv-int/9508005

[24] Svinolupov S I and Habibullin I T 1996 Integrable boundary conditions for the multicomponent Burgers equation Matem. Zametki $\mathbf{6 0} 888$ 